\title{
User-Oriented Design of Active Monitoring Bedside Agent for Older Adults to Prevent Falls
}

\author{
Norihisa Miyake ${ }^{1}$ (D) . Shouta Shibukawa ${ }^{2} \cdot$ Harue Masaki $^{3} \cdot$ Mihoko Otake-Matsuura $^{1}$
}

Received: 14 August 2018 / Accepted: 12 June 2019/ Published online: 5 July 2019

(C) The Author(s) 2019

\begin{abstract}
A small bedside agent for preventing falls has been developed. It talks to a person on a bed to prevent them from getting out of bed abruptly, until a care worker arrives. This paper describes the user-oriented design process of the agent system. The development process involving users, such as nurses and caregivers, as well as older adults is described. First, hardware design, such as the outer shape, size, and function of the agent was reviewed by nurses and caregivers, mainly from a safety viewpoint. The prototype agent incorporating improvements based on their opinions was used experimentally by older adults after several review processes. Second, the software design of the agent, such as the content of voice call, was studied through multiple experiments to improve its acceptability. Lastly, the integrated model was introduced into care facilities and hospitals to investigate the practical serviceability of the system.
\end{abstract}

Keywords Bedside agent · Fall prevention · User-oriented design · Assistive robotics

\section{Introduction}

The number of older adults is reported to be increasing, which is consistent with rapid aging of the society in many countries, including Japan, which is already called a "superaged society" [1]. Consequently, the number of older adults with physical or mental disabilities and needing care or assistance is increasing, because of their declining physical ability and increasing frailty due to aging. Apparatuses to assist the daily living activities of older adults are strongly needed [2] to reduce the number of people who require manual nursing care. Replacing human support with apparatuses such as robotic devices to assist disabled older adults with daily living activities is expected to

Norihisa Miyake

norihisa.miyake@ riken.jp

Mihoko Otake-Matsuura

mihoko.otake@ riken.jp

1 Center for Advanced Intelligence Project, RIKEN, 1-4-1 Nihonbashi, Chuo-ku, Tokyo, 103-0027, Japan

2 Paramount Bed, Co. Ltd., 2-14-5 Higashi-suna, Koto-ku, Tokyo, 136-8670, Japan

3 Graduate School of Nursing, Chiba University, 1-8-1 Inohana, Chuo-ku, Chiba, 260-8672, Japan substantially contribute to the smooth functioning of superaged societies.

As the word "bedridden" implies, getting out of bed is one of the most important daily living activities to maintain a person's physical functions. Commonly, however, accidents such as "falls" which can most likely cause physical handicap and even worsen a person's symptoms, occur near the bed where an older adult spends considerable amount of time during the day and night [3]. Numerous research and development efforts related to assistive devices have been undertaken to solve such problems [4].

Falls among older adults occur mostly because of their poor ability to maintain dynamic balance, not because of reduced muscle strength. Consequently, "fall" accidents are listed as the most frequently occurring incidents in hospitals, at least in Japan, and the number of such incidents has been increasing because of an increase in the average age of hospital inpatients $[3,5,6]$.

Building a safe bed environment, one of the major components of the ambient assisted living concept [2], is imperative in hospitals and care facilities. One of the directions of such efforts is the research and development of a monitoring system.

Pressure-mat-type sensor devices [7] are widely used for monitoring the posture of a person, although they have a few drawbacks, including poor reliability. The use of TV 
cameras or microphones for monitoring a person's behavior is not recommended because of privacy issues, although cameras can precisely detect the movement of a person on or near a bed. Studies on the use of infrared sensors, ultrasonic sensors, and force sensors to detect the movement or posture of the person on a bed have been carried out as well [810]. However, these systems assist caregivers, that is, nurses and care workers, by relieving their burden by providing information even when the person being monitored is not near the caregivers.

Systems that respond to the action of older adults exist, and such systems reach out directly to the person. They are intended mainly for communication or therapeutic purposes, not for preventing falls [11, 12]. In addition, systems called social robots are used in environments with older adults. The major objective of these systems is to increase the opportunity for communication by initiating conversation [13, 14].

The authors' ultimate motivation in this study is to develop a monitoring system that is both friendly to a person on or near a bed and beneficial to caregivers. From this viewpoint, the concept of the system is set as an active system that reaches out to a person; therefore, a robotic agent is selected as one of the best-suited candidates for this purpose because of affinity with the person being cared for. Such a human-friendly agent that behaves softly with the person receiving care is presumed to provide a feeling of security.

This study presents a specific vision of the aforementioned concept, especially the desirable and appropriate form and aspect of a system based on the concept, and clarifies its acceptability through the development and testing of such an agent system.

In this paper, an outline of the development method is given and the system development process is explained. In addition, a brief outline of the system is presented and the feasibility of the system is evaluated through verification experiments.

\section{User-Oriented Design Methodology}

\subsection{Research Objectives}

There are various approaches to prevent patients' falls. These approaches can basically be divided into two categories, namely physical methods and mental methods. One typical example of the physical methods is to put a fence or siderail(s) around the bed, so that the patient cannot leave and fall from the bed physically. However, this type of a method is considered as the "medical restraint", since it deprived the patients of freedom, even for the patients with mental illness. Therefore, such a method is not recommended to be utilized in hospitals and care facilities, at the government level in many countries including US and Japan [15-17].

Thus, the physical methods, securing safety at the expense of patients' freedom, are considered undesirable, and not suited for the solution to prevent falls. For example, even a device like a fence which is actuated and set in place only when needed, is considered restraint because the device physically blocks the patient's intention of getting out of the bed, although engineers tend to prefer this type of seeds-oriented approach. Also, it would be potentially hazardous when there were some moving parts, that move automatically, independent from the patient's intention, in the vicinity of a patient in a bed. As a result of the above discussion, the authors made it a policy to solve the problem by not applying the physical means, and utilizing only the mental means, or non-physical assistive means, when designing the system.

The bedside agent system developed here, which is intended to be used in hospitals or care facilities, monitors a person's behavior to provide caregivers with information in addition to providing a voice-call-based function for preventing or postponing behaviors that lead to falls and similar accidents. That is, the main feature of the system is that physical means are not used to prevent falls. Instead, falls are prevented by human caregivers' physical assistance. The agent system provides "talks" or voice calls, which remind the person to rethink and suppress behaviors leading to falls, as human caregivers provide conventionally. The burden of caregivers would be minimized if the bedside robotic agent system can perform the caregiver's function of preventing the person from acting dangerously, even though the agent is not equipped with physical means to prevent falls. The quality of nursing care could be greatly improved with the help of this type of system.

\subsection{Basic Concept}

The main objective of the agent system is to provide assistance that contributes to reducing fall accidents without using physical means. Currently, human caregivers or nurses watch a patient who is at risk of falling and offer assistance when necessary. Usually, a "nurse call system" is used when a patient needs help. It is the top-priority task for nurses to respond to nurse calls and to go to the patient's ward as soon as possible. Thus, fall accidents can be prevented if the nurses can visit the patient's side before the patient leaves the bed. Therefore, the ability to postpone the patient from leaving the bed, at least by the time the nurses reach the ward, is quite beneficial [18].

From this point of view, the system detecting the patient's activity that leads to leaving the bed, launching the nurse call, and allowing the patient to postpone leaving the bed 
with the voice calls as long as possible would be very helpful. Such a system would be beneficial for nurses as well as for patients, even though the effect of the postponed time span is limited, because the chances of a patient falling as a result of unattended movement can be reduced if the nurses can reach the ward before the patient leaves the bed.

In summary, i) if a patient's action to leave bed can be postponed, then ii) the chance for the nurses to visit the patient before the patient leaves bed can be increased, thereby iii) reducing the number of fall accidents. The basic concept of the bedside agent system can thus be expressed as "buying time for nurses to come to the patient's aid".

\subsection{Design Methodology}

Various types of assistive devices for older adults are available, although not all of them are truly applicable in real scenarios. The major reason for such a situation may seemingly be because a large number of such devices were developed following the seeds-oriented approach. The needs-oriented approach, by contrast, has been used rarely because it is time consuming and is associated with many difficulties such as real needs extraction and appropriate technology matching.

Notably, when carrying out this type of research, following the needs-oriented approach or the user-oriented design approach is essential to realize user-friendly systems for older adults to the extent possible. The flowchart in Fig. 1 shows the user-oriented procedure adopted in the present work for system development and implementation, although the processes indicated by dotted lines are incomplete at present.

Lessons learned from the development are summarized as follows. First, interviews with nursing staff at hospitals and staff at care facilities were conducted. This is seemingly an obvious first step in the user-oriented approach to extract system prerequisites. However, the information extracted from nurses and care staff is not "what IS needed," but "what is NOT good." Such denial and repudiation are most likely the common responses from the staff, and extracting the real needs from the older adults themselves is even more difficult.

The important activities in the user-oriented design approach are i) to carefully masticate and analyze the interviewed results, ii) to form hypotheses and make preliminary prototypes, iii) to re-interview the users or persons concerned, iv) to feed back the interviewed results to refine the hypotheses, and v) to repeat the series of this plan-do-check-action (PDCA) processes several times to realize what users truly need, to meet the real requirement of the field of nursing and care.

Because the initial requirements described by the intended users were implicit, explicit requirements were

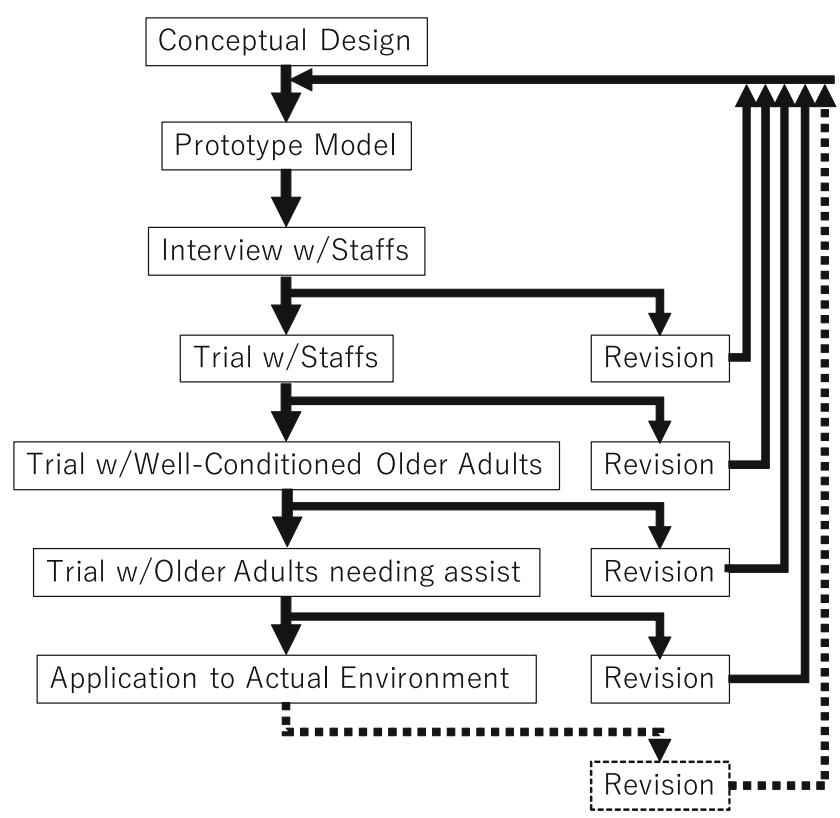

Fig. 1 Process for user-oriented design

revealed incrementally through the recursive process of prototyping and reviews. In addition, approval from the ethical review boards is imperative at each step of the field interviews and test phases.

In the following sections, the steps of the development process are briefly described, along with an overview of the agent system.

\section{System Outline}

\subsection{Interviews with Nurses/Caregivers}

First, interviews were conducted to clarify the needs of nurses and caregivers. The most prevalent problem was assumed to be "fall" incidents near patient beds, as explained previously, and the interviews focused on the means to prevent such incidents from occurring. The responses gleaned from the interviews show that the means for preventing falls are i) constant periodical patrol rounds to assess the patients' condition and state, ii) talking to the patients during the patrol rounds to make them feel secure, iii) letting the patients be active in the daytime and sleep well during nighttime, iv) encouraging patients to go to the toilet before sleep to reduce the need to leave the bed for visiting the toilet during the night, and v) encouraging, through the nurse call system, the patients to stay in bed so that the nurses can arrive at the patients' ward before the patients leave bed. Notably, all of these measures are indirect, nonphysical-contact methods. 


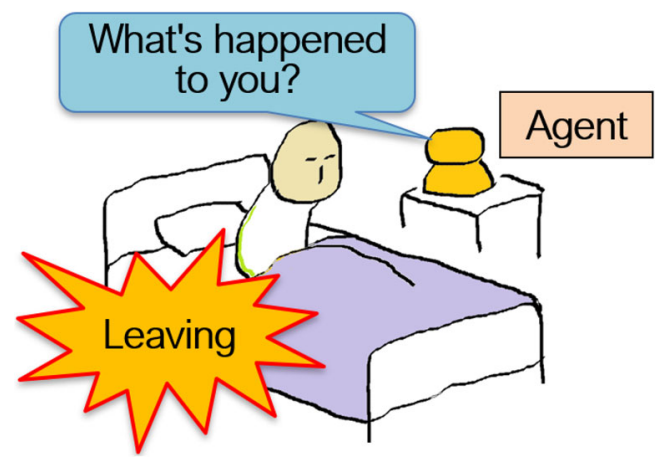

Fig. 2 Image of bedside monitoring agent

The aforementioned needs lead to the idea of a noncontact "voice call" type active monitoring agent system, as shown in Fig. 2. The agent system is expected to substitute the staff by voice calling the patient in the bed to ensure patient safety and reduce the burden on the care staff; the realization of this expectation is contingent on whether the system can talk the patient out of getting out of bed until the care staff arrive at the scene. The voice call is also considered very important in nursing/caring environments to provide a trigger for the patients to communicate and make their intention known to the staff [19]. The expected users of the system are patients who can notice voice calls and are at a high risk of falling if they attempt to get out of bed unassisted.

\subsection{System Requirements}

Nurses have various missions to perform and cannot remain at all times at the bedside of patients at high risk of falling. Although nurses are expected to prevent fall accidents, this task cannot be done unless they are near the patients. Therefore, as described in the previous section, a bedside agent system that can postpone the patient's action to leave bed until the nurses arrive at the patient's side would be useful [18]. According to Japanese standards for hospital care, a nursing unit is generally set to monitor 40 patients, where 4 to 6 nurses are assigned during daytime and at least 3 nurses are assigned during nighttime, depending on the severity of the conditions of the patients in the ward. For the system designed here, the longest length of a nurse's activities from the nurse station to a patient's room is set to 25 meters, considering the standard layout of a hospital's general ward, where the nurses' station is located near the center of the ward [20]. The duration required for the agent to postpone the patient's leaving bed can then be calculated as $15 \mathrm{~s}$, which is the duration for nurses to arrive at the farthest-located ward assuming that the nurses' quick walking speed is $6 \mathrm{~km}$ per hour. Note that the time required for a patient to move from supine position to sitting square

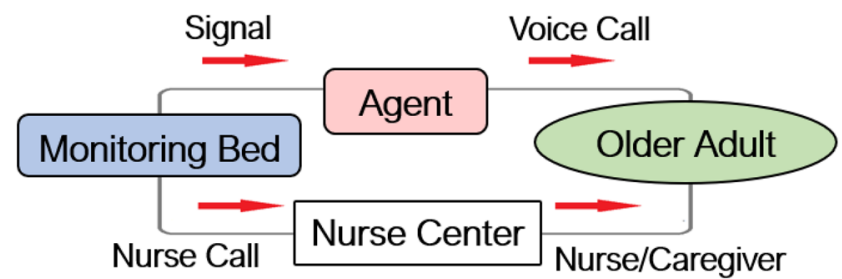

Fig. 3 System outline

position, which is additionally needed for a patient to leave a bed, is not included in this time. Therefore, the agent system is beneficial if the system can continue to attract a patient's attention and allow the patient remain on the bed for the time period of $15 \mathrm{~s}$, for instance, to theoretically minimize unattended fall accidents.

\subsection{Outline of System Configuration}

The active monitoring agent system with a voice call, shown in Fig. 3, consists of i) a sensor system to detect the motion of a person on a bed, and ii) an agent robot for voice calling. The sensor system is connected to the nurse call system to let nurses/caregivers know about the person's behavior when assistance is needed. The sensor system's output is also monitored and the voice call of the agent is activated when such help is needed.

\subsection{System Implementation Procedure}

Evaluation of the developed system at hospitals and care facilities is the most important process in useroriented design. The following steps are related to system implementation and verification of system serviceability.

Step 1: Functionality and safety evaluation of the system (Adequacy for medical/care wards)

Step 2: Evaluation of human-agent communication (Acceptability from older adults)

Step 3: System verification in real environment (Applicability for practical service)

\section{Extracting a Person's State on a Bed}

\subsection{Bed Sensor Systems}

According to statistical data, "falls" are most likely to occur when a person gets out of bed [3, 8]. The states of a person on a bed are categorized with respect to the motion sequence of a person leaving the bed as i) supine (dorsal) position, ii) long sitting position, and iii) sitting square (at bed edge), as shown in Fig. 4. These categorized states represent the 
Fig. 4 Detection of a person's state
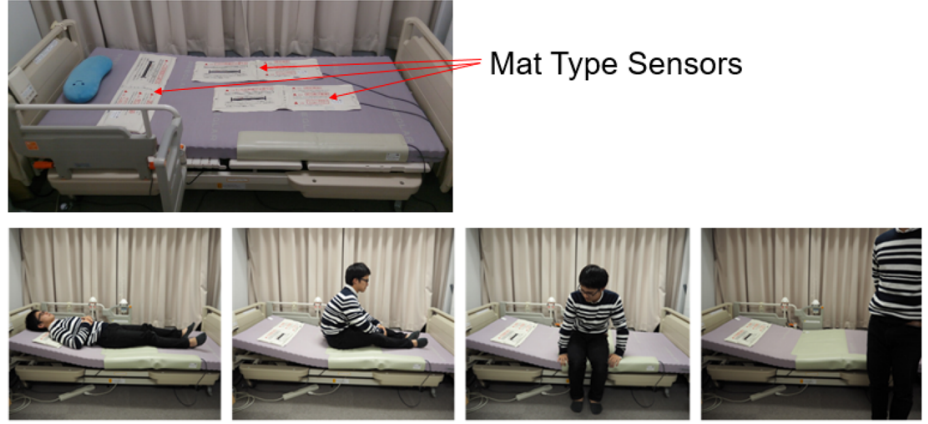

Position: Supine

Chest/Back

Waist/Hip

Edge

1

1

0
Long Sitting

0

0
Sitting Square

Left

0

1
0

0

0

(1: pressed, 0: free)

typical phases that lead to standing up from a bed and can thus be regarded as predictors of whether a person intends to get out of bed.

A system called "Getting-out-of-bed CATCH" is installed in hospital beds and in care beds. In this system, load sensors are equipped with actuators for back lifting and frame lifting $[9,10]$. The system can detect the aforementioned three phases of the process of getting out of bed, along with the output pattern and time transition of the sensors, and is widely used in hospitals and care facilities.

Although the last state before getting out of bed is "sitting square" at the edge of the bed, "long sitting" is the first state in getting out of bed, and it can thus be treated as forestate information. Providing such information in advance to caregivers is important for preventing certain types of patients, such as the ones agile in motion, from falling because the nurse or caregiver can be alerted to visit the patient before he/she gets out of bed.

\subsection{Sensor System Design}

The aforementioned system is useful and suitable for installation in hospitals and care facilities as long as the bed is set at a fixed location. However, transporting the bed every time to the facility where the experiment is conducted is not easy. In such a scenario, a system comprising multiple mat-type sensors is used to ensure portability, as shown in Fig. 4. The sensor mats are aligned at the chest/back part, waist/hip part of a person on the bed, and the edge part of the mattress on the bed. Thus, the three phases of the process of getting out of bed can be detected, as shown in the figure, to activate the nurse call as well as the agent's voice call as explained in the following sections.

The state or the posture of a person on a bed was determined with a series of mat sensors' data, which were measured every $0.5 \mathrm{~s}$; and the accuracy of the detected state when 6 data points $(3 \mathrm{~s})$ were used for the recognition was 81 to $93 \%$, varying from person to person. The false recognition errors that occurred depended mostly on the person's agility. The applicability of the sensor system for field tests was thus demonstrated through preliminary experiments.

\section{Bedside Agent}

\subsection{Requirements for Bedside Agent: First Design}

First, the design policy of the robot agent from the viewpoint of safety requirements was examined by following the general risk assessment method [21]. The bed can be moved to other rooms in the event of an emergency, and the use of wireless communication systems is restricted, especially in hospitals, where the electromagnetic compatibility characteristics of the system are important. On the basis of these requirements, the agent system was designed as follows: i) the controller was placed beneath the bed, ii) the agent was connected to the controller by wire, and iii) the agent was designed to be placed at the bedside table.

The agent robot should preferably give off a humanfriendly feeling or exhibit a high degree of affinity for a person on a bed. The size of the agent was initially set to $100-200 \mathrm{~mm}$ on one side; it was designed with a round shape without protrusions or sharp edges and was lightweight. A robot is known to attract a person's attention when it moves [22, 23]. Therefore, the agent robot was designed to have moving parts to attract the patient's attention, although the degree of freedom of movement should be as low as possible to reduce any potential for hazard as well as to increase system reliability. In addition, the mechanical structures of the movable parts, such as joints, were designed to prevent any body parts from getting caught in them. Prevention of electrical shock and 
waterproofing of the mechanism were considered in the design stage.

The first prototype of the bedside robot agent based on the aforementioned considerations, which the authors named "Side-Bot", is shown in Fig. 5. This robot has two degrees of freedom: vertical and horizontal head shake (expressing yes and no) capability.

\subsection{First Evaluation by Nurses and Care Workers}

The first prototype of the robot agent was brought into the medical wards of the rehabilitation and orthopedic surgery department of a hospital to be evaluated by nurses and certified care workers. The evaluations were made mostly from the viewpoint of patient safety, and refinements to the design were made until evaluations indicated that no possible hazards remained.

"Side-Bot" was introduced in a simulated room of the medical ward and demonstrated to teams of medical staffs, each consisting of three to four persons. The robot's voice calls and motion were demonstrated in a scenario in which a typical ward patrol round was simulated. The performance of the robot was evaluated by three different teams after each demonstration.

The points of evaluation emphasized by the authors were i) safety of robot movement, ii) the possibility of the robot falling when placed on or near the bed, iii) the safety of the robot when it is dropped, iv) feeling of security (shape, face design), v) robot size (for placement near or on the bed such that it is noticeable to the person), vi) placement location (negative effect on nursing and caring task), vii) voice call (method and contents), viii) sound volume and directional characteristics, ix) electrical shock and waterproofing quality, and $\mathrm{x}$ ) overall safety.

As a result, the major problems to be solved for actual installation of the robot in the ward were identified as follows: i) movable parts are not desirable from safety viewpoint, even though they are designed to prevent body parts from being stuck in them; ii) possibility of dropping remained; iii) possibility of chippage and breakage when dropped remained; iv) face design is too realistic; v) size should be smaller; vi) no space on the bedside table because of personal belongings, nor by the person on the bed because of the back-lifting function of the bed; and vii) motor sound should be quieter to avoid distracting other patients in the same ward.

These results show that the agent is not necessarily a robot that moves. Moreover, the expected situations in which the agent is most needed are mornings and nights, when the staff are the busiest and it is difficult for the agent to attract attention even though it moves because it is difficult to observe in the dark, especially during nighttime. On the basis of this result, luminescence, instead of motion, along with voice and sound, were considered more appropriate cues from the agent.

The results of evaluations by the staff revealed additionally that the human-like face design does not always give a human-friendly impression, especially when the robot is placed near the person all the time. A simple doll-like design is more suitable in the target environment, especially when the agent is not in use.

In addition, it was revealed that the agent should not be placed on the bedside table because the table holds various personal belongings and medicines and because there is no space for the agent in most cases.

\subsection{Second Design and Evaluation}

"Side-Bot" was then improved based on these evaluation results, and the second design of the agent named Prototype II (Fig. 6), was fabricated. The size of the agent was $80 \mathrm{~mm}$ on one side. This version i) had no motorized moving parts, ii) was equipped with an arm/hanger wire so that it can be suspended from the side rail of the bed, and iii) was lightweight because of the absence of motors. The design was inspired by a Japanese Kokeshi doll, and it was equipped with LEDs on the cheeks of the face.

The second prototype was evaluated by the medical staff. The results of the evaluation were mostly good and acceptable, except for the fact that the agent was too small and some patients could accidentally put it into their mouth.

Therefore, another refinement, that is, the size of the agent was increased to $120 \mathrm{~mm}$ on one side to ensure that the agent was suitably large. Because the agent cannot express emotions by bodily motion nor attract attention by moving, it was designed to i) address the person being cared for by name, and ii) emit light from LEDs, both before talking, to draw patients' attention.

\subsection{Evaluation with Community-Dwelling Older Adults}

A preliminary experiment with 16 university students and 4 community-dwelling healthy older adults (age 67 to 77) was conducted before the system was introduced into an actual medical ward. In this experiment, the mechanism and function of the second prototype, along with the contents of the agent's speech, were evaluated.

The major points investigated included whether the participants noticed or understood the agent's speech, any preference between a male voice vs. a female voice, and any feeling of stress. The transcripts of a few of the utterances of the agent, which are voiced using synthetic voices, are listed in Table 1. The experiment was conducted in a facility's meeting room environment, and each participant, 
Fig. 5 Side-Bot Prototype I

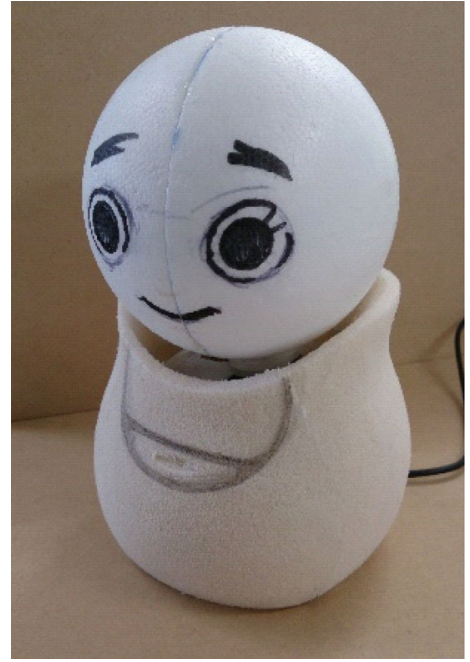

(a)Overview

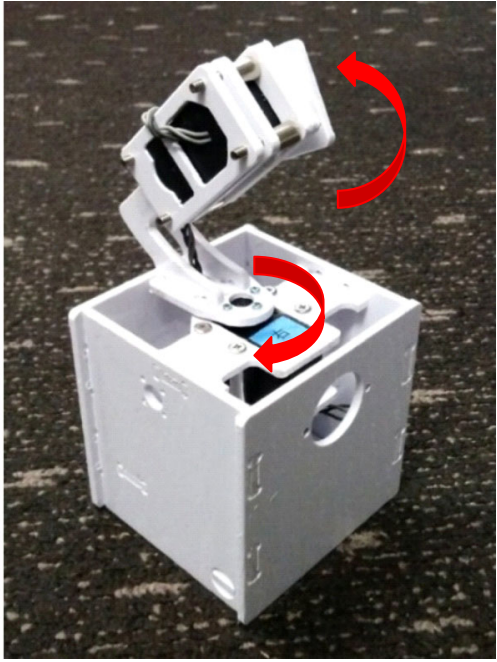

(b)Internal Mechanism one at a time, was introduced into the room to perform the experiment. Two agent robots were set on a table, and the contents and timing of the agent robots' speech were controlled manually by an experimenter not seen from the participant. The experimenter starts the robot agents to make speech, once the participant has been settled and gotten used to the room environment. There was an observer as well, also not seen from the participant, to record the response of the participant.

The experimental results were evaluated using objective measures, observed from other peoples' perspective, and subjective measures, obtained as the feedback from the participants. The objective measures were an attention score (estimated by the participant's line of sight: gazing at the robot when the robot is talking) and a recognition score (measured by the adequacy of the participant's answer to a question posed by the agent). The subjective measures were the participants' visual analogue scale (VAS) answers to the questionnaires regarding audibility (self-evaluation about the understanding of the agent's speech), unnaturalness of the conversation, and stressfulness. The objective scores were measured by an observer who monitored the response of the participants. This human-based method, rather than recording the scene using a video camera, was used for consideration of the privacy of the participants.

The results shown in Figs. 7 and 8, as examples of the experimental results, indicate that the recognition score and audibility were better when the agent first addressed the participant by name. However, a significant difference in this regard was observed only with students because

Fig. 6 Side-Bot Prototype II
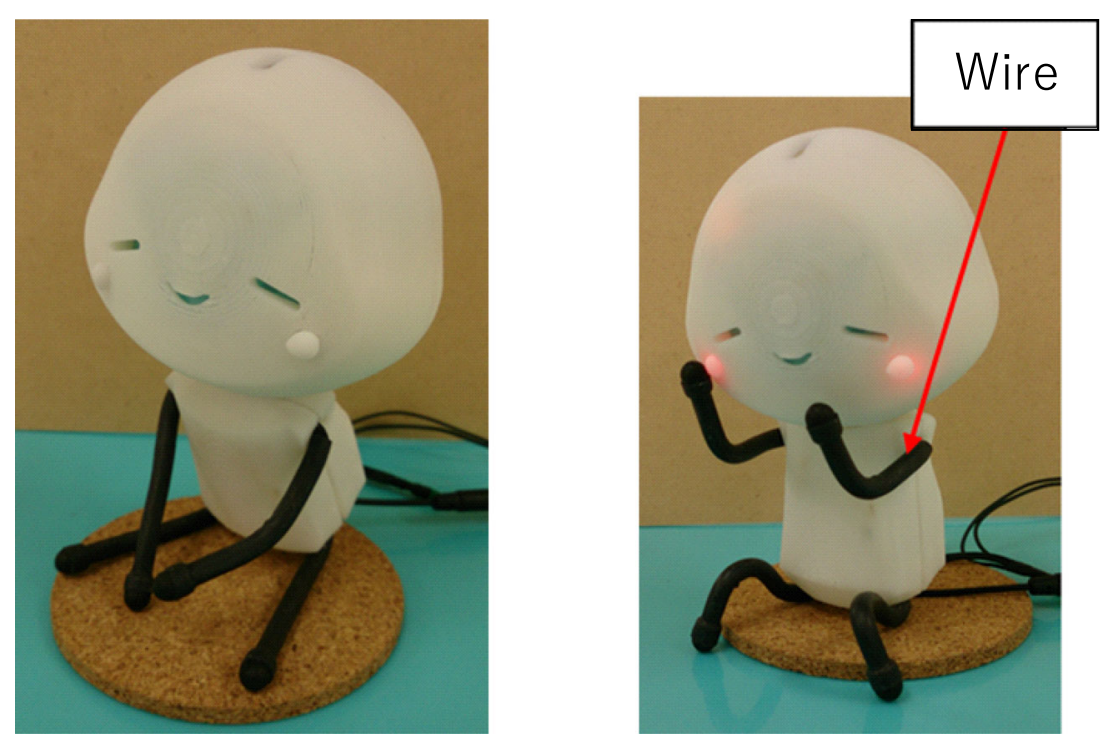
Table 1 Example of robot speech

\begin{tabular}{lll}
\hline Name call & Voice type & Speech content \\
\hline No & Male & $\begin{array}{c}\text { Would you tell me a word } \\
\text { beginning with "N"? } \\
\text { Would you tell me the name of } \\
\text { something yellow? }\end{array}$ \\
& Male & Where would you like to travel? \\
Yes & Female & Mr(s)., would you tell me a \\
& Mord beginning with "M"? \\
& Mr(s)., would you tell me the \\
& Female & Mr(s)., what is your favorite \\
& & animal?
\end{tabular}

the number of older adult participants was limited. The difference in recognition scores with or without LEDs was found to be not significant, although slight gaps between the average data are evident in the figure in all cases.

The results also showed that the synthetic voices were not always easy to hear. Nevertheless, female voices were preferred according to the VAS evaluation, although there was not a significant difference in terms of audibility. One case was reported as stressful because the patient found the voice difficult to hear.

Additionally, some of the test subjects commented that visibility of the light emission was insufficient and that the stability of the agent's body was unsatisfactory. The third prototype was then designed on the basis of these findings.

\subsection{Third Design and Evaluation}

The third prototype model, shown in Fig. 9, was designed to solve the aforementioned problems. That is i) the external shape was designed to be squeezed slightly to provide a feeling of comfort, ii) the center of gravity of the agent was lowered by changing the position of the speaker to increase stability, and iii) LEDs were relocated to irradiate the head portion from the cheek parts.

\section{Content of Voice Call}

As described in the preceding section, the authors optimized the hardware design of the agent. However, the content of the voice call, or what the robot must utter, must be examined to achieve the objective of the agent, which is to attract the attention of the person on the bed and to ensure that the person remains on the bed longer. An experimental study of the content of the voice call or speech is described in this section.

\subsection{Scenario-Based Talk}

Achieving a dialog with an agent requires voicerecogni-tion technology, discourse-comprehension technology, conversation-generation technology. These technologies are not easy to implement when the dialog with the agent and a person is required to be realized in a "natural" manner. One of the reasons for this difficulty is that speech recognition and understanding the intentions of older adults is not easy.

The authors developed a technique based on "scenario prediction-based dialog", which does not depend on voicerecognition technology. It was found from the interviews that, to the extent possible, nurses try to voice-call patients by using inquiries that can be responded to with simple "yes" or "no" answers. This technique avoid causing panic and allows the nurse to understand the patient's request correctly. This setting resembles the dialog system, where questions have limited types of expected answers [24, 25], with the difference being that the type of expected answer is unique.

The robot agent developed herein was programmed to speak in phrases such that the responses to the phrases are basically predictable. Thus, the answers to these responses could be preprogrammed in most scenarios, without the need for recognition. The person giving attention to the agent can feel as though he/she is having a conversation, even though the agent is speaking according to the scenario by simply controlling the timing of its utterances. That is, the agents' utterances, after the questionings, were chosen to provide "back-channel feedback" type utterances, as shown in Tables 2 and 3, that were not affected by the person's answers.

\subsection{Dialog-Style System}

Ishiguro et al. [26] has shown that a person begins to have a feeling of involvement, or participation awareness, by simply looking at two agents having a dialog according to a scenario. This feeling of dialog is important for ensuring that the communication between an agent and a person seems natural and acceptable. Therefore, a system comprising a pair of "Side-Bots" was used in the following experiments, as shown in Fig. 9.

\subsection{Experiments}

The proposed system was introduced into a medical ward for experiments with patients after approval was obtained from both our institution's ethical review board and that of the hospital. Experiments with 12 older adults at a care facility revealed information about i) the effect of attracting attention, ii) audibility and sense of comfort of patients to 
Fig. 7 Recognition scores (objective evaluation)
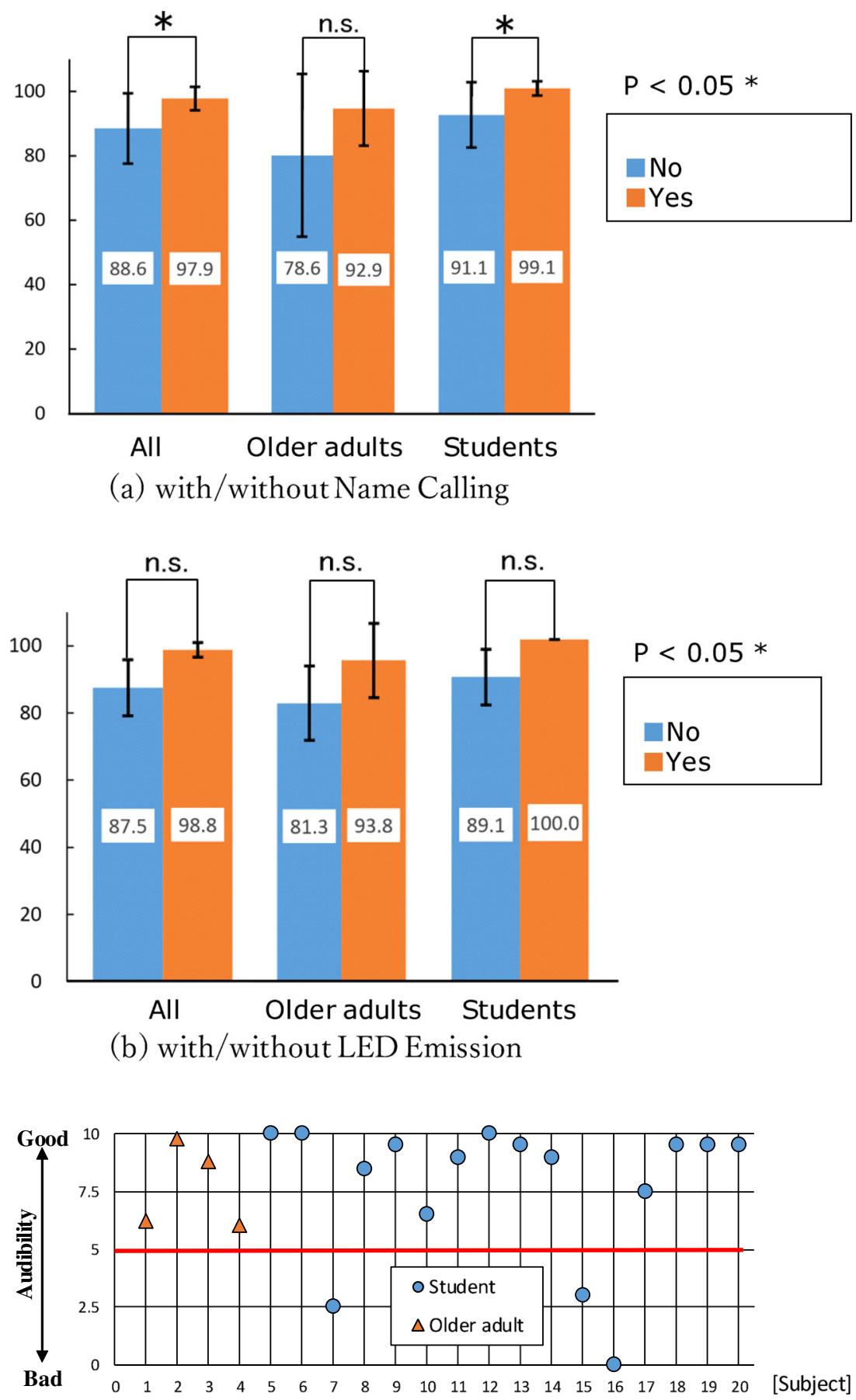

(a) Name Calling

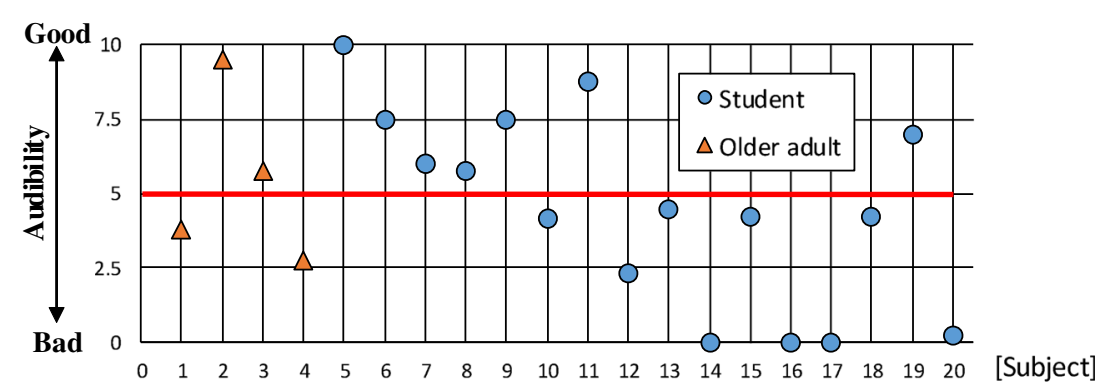

(b) LED Emitting (a) with/without Name Calling

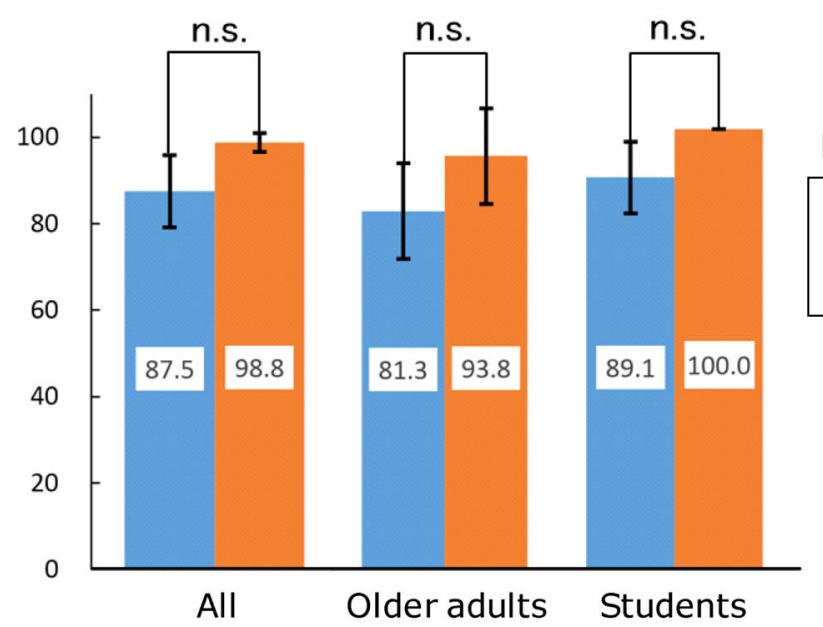

(b) with/without LED Emission

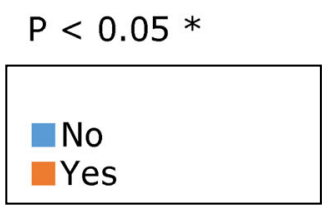

$P<0.05 *$

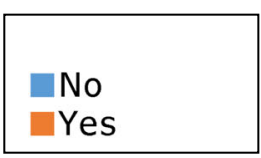

Fig. 8 Audibility scores (subjective evaluation) 
Fig. 9 Side-Bot Prototype III
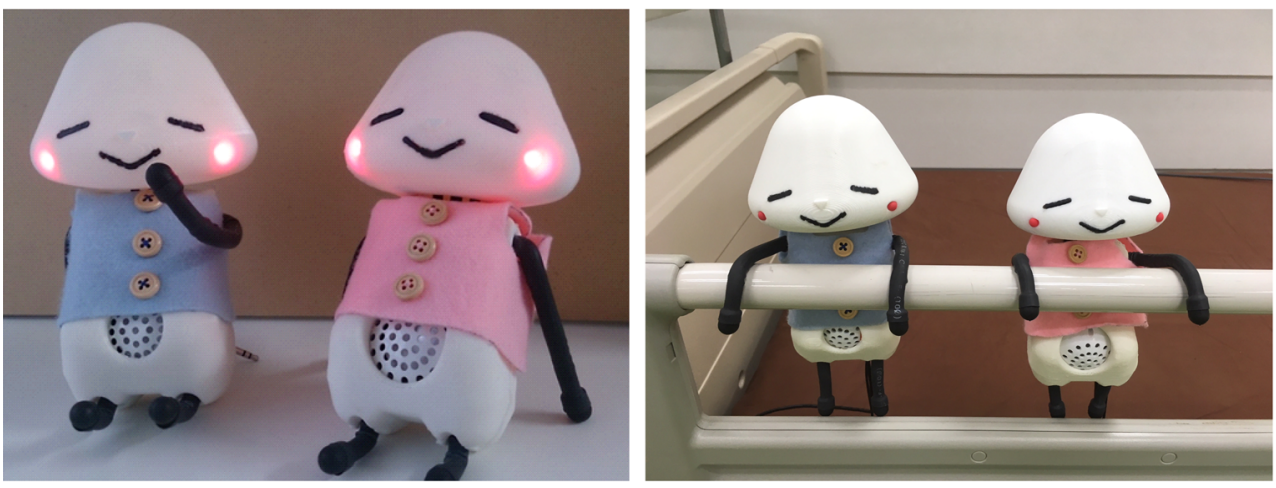

contents of the agent's utterances. The experiment duration was approximately $30 \mathrm{~min}$, and the effect, audibility, and comfort were evaluated by observation and based on a questionnaire administered to the participants.

The agent system consisting of two "Side-Bots" was operated manually from a remote location not visible to the participants. The reason for using this configuration was to find the patients' responses and to choose the appropriate contents of the agent's utterances. The agent's utterances were chosen to extract expected responses from the patients through analysis of the results of this experiment.

The experiments comprised three conversation parts, each of a different type: i) word repetition (type 1), ii) everyday conversation with an agent (type 2), and iii) everyday conversation with a pair of agents (type 3), as shown in Fig. 10. In type 2, robot A is programmed to reply to the participant's answers, whereas robot $B$ is programmed not to reply, to observe differences in the participant's impressions. In type 3 , robots $\mathrm{A}$ and $\mathrm{B}$ converse dialogs according to the scenario, as shown in Table 2, and then ask a question to the participant and respond to the participant's answer. Here, multiple scenarios (five to twelve, depending on the type) were prepared for each conversation type to avoid dispersion of the experimental results. The average time durations of each of the three types of scenario were approximately 1,5 , and $15 \mathrm{~s}$, respectively, based on the discussion in Section 3.2.

\subsection{Results}

The experimental results were evaluated using objective and subjective measures, as described in Section 5, and the preferences of robot $\mathrm{A}$ and $\mathrm{B}$ were added along with other indexes, such as attention/recognition scores and stressfulness.

The rate at which attention was attracted by the added LEDs and the name call before the conversation, as measured by the participant's line of sight, was found to be approximately $70-90 \%$ on average (50-100\% depending on the participants), as shown in Fig. 11. This result reveals that the LED and name call did attract attention, irrespective of the type of conversation. The rate of recognition, as measured by the participant's response words, was found to be 40-100\% depending on the participant, and conversation type 1 was less recognizable, presumably because words were spoken abruptly.

The result of the questionnaire showed that $75 \%$ of the participants did not feel stress. The remaining $25 \%$ felt some stress, mostly because of difficulty in hearing the agent's artificial voice. The impression of two agents (robot A and B) did not much differ, which means that whether the agent replied or not did not affect the preference for agents.

Regarding the naturalness of the conversation, $70 \%$ answered that the conversation felt natural, which indicated the practicality of the scenario-based dialog method, although $30 \%$ felt otherwise on the points such as the conversation interval. This issue warrants further investigation.

In addition, the results of this experiment show that the scenario-based conversations could attract participants' attention and that their attention was retained so long as the agents continued to speak. This means that the participants' attention can be maintained up to $15 \mathrm{~s}$, when the Type-3 scenario was employed. The major purpose of this agent system is to let the person on the bed rethink and suppress behaviors leading to falls until a nurse arrives at the person's side. Thus, the dialog-style conversation not only creates a natural and acceptable feeling but also continues for the longest period among the three types and is most effective for retaining a person's attention. Thus, it is the most suited

Table 2 Example of robot dialog

\begin{tabular}{ll}
\hline Speaker & Speech content \\
\hline Robot A & It's hot today, isn't it? \\
Robot B & Yes, it's hot today. \\
Robot A & How about you, how do you feel? \\
Subject & (Me too! It's really hot today.) \\
Robot B & Thank you for answering We are happy!
\end{tabular}


Fig. 10 Conversation types in the experiment

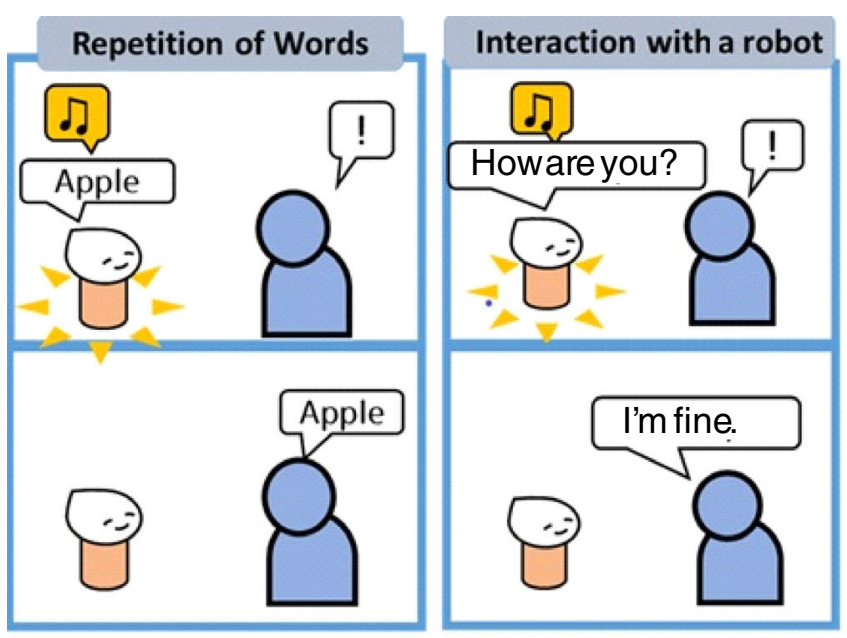

(a) Type-1

from the viewpoint of developing the content of the agent's utterances.

\section{Experiment for System Verification}

Verification of the system in a real hospital and care facility with continuous system operation over an extended duration is the most important phase of this type of system development. The sensor system, bedside agents, and dialog contents were integrated into a fully automated active monitoring bedside agent system. The timing and content of the utterance, in the following experiments were determined and controlled automatically by the status change of the

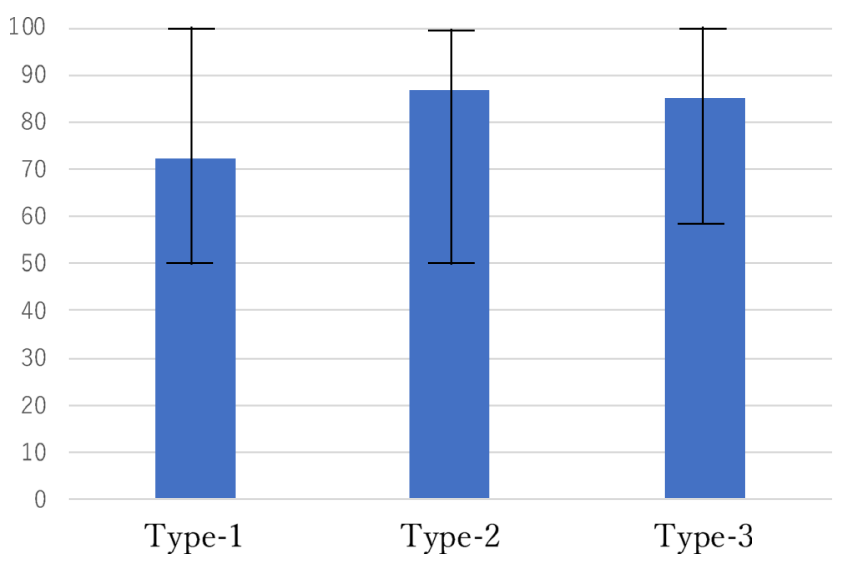

Fig. 11 Attention score vs. conversation type

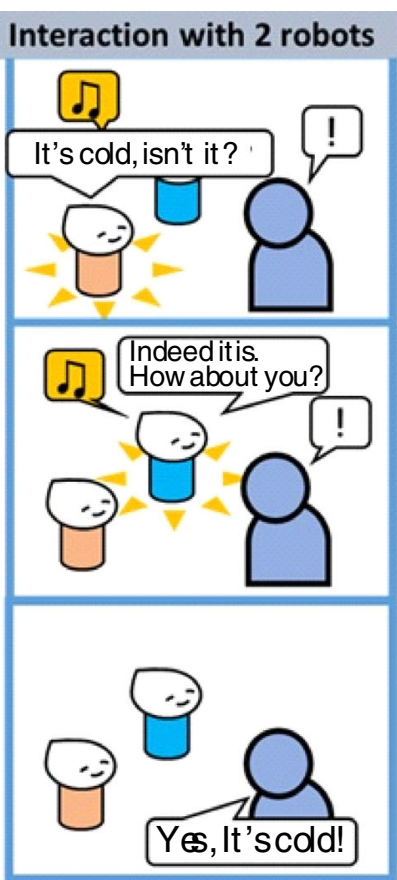

(c) Type-3 (b) Type-2

sensor system described in Fig. 4, which indicated the patient's posture change.

Positive results were obtained through preliminary trials, but additional experiments need to be conducted. An outline of this phase is described in this section. The experimental procedure was screened and approved by both the ethical review committee of our institute and that of the facility.

\subsection{Preliminary Experiment with Students}

An experiment with 11 students was conducted. The experiment duration was set to $240 \mathrm{~min}$, and system operability was studied. Sounds were recorded at intervals of $60 \mathrm{~s}$ after the agents started talking. An example of the talk is summarized in Table 3. The scenario was controlled by the sensor output which indicated the posture of the patient, as shown in Table 3. Also, the contents of the scenario were chosen such that they were not strongly affected by the answer of the patient, whether it be yes or no, to provide a "back-channel feedback" type utterance, as shown in the table. The major evaluation points were safety and acceptance of the system. Function, response to the agents' speech, response time, time duration of the attention, stress and fatigue were examined as well. Here, the participants' behavioral response and utterances were measured using sensor mats, as explained in Fig. 4 along with a voice recorder for evaluation purpose. The stress and fatigue were evaluated using participants' subjective answers to the questionnaires. 
Table 3 Example of the scenario

\begin{tabular}{lll}
\hline Position & Speaker & Speech content \\
\hline Supine & Robot A & Want to sleep? Good night. \\
& Robot B & I'll keep quiet. Good night. \\
Long sitting & Robot A & Mr(s)., did you sleep well? \\
& Subject & - \\
& Robot B & We are happy if you could \\
& & talk to us. \\
Sitting square & Robot A & Would you like to go to the \\
& & bathroom? \\
& Subject & - \\
& Robot B & We are waiting for you. \\
\hline
\end{tabular}

The results show that i) "go in one ear and out the other"type topics were useful, along with questions that need to be answered, for minimizing stress and fatigue, and that ii) the dialog method elicited responses from the participants, although frequent and short talks caused discomfort to the participants. Also, it was found that the participant's attention was retained so long as the agents continued to speak. The scenarios and their timings were readjusted on the basis of the results.

\subsection{Preliminary Experiment at a Care Facility}

A 48-hour experiment was conducted with two older adults each living alone and two residents of the care facility to verify system utility. The experimental scenario, experimental conditions, evaluation points and methods

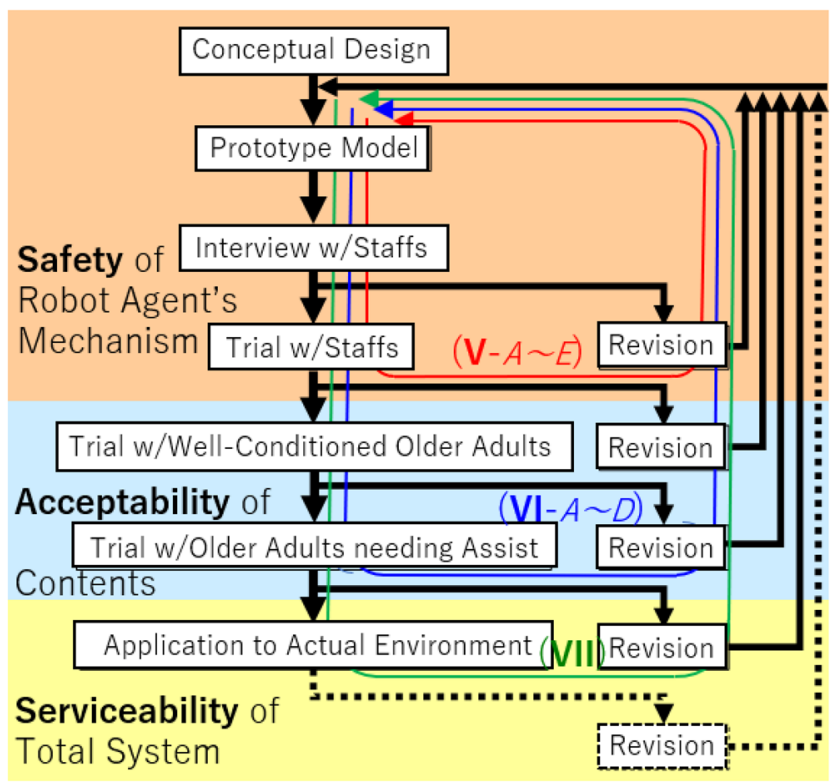

Fig. 12 User-oriented design process for Side-Bot were the same as those in the experiment involving students. Video cameras were not used because of consideration of the participants' privacy, although microphones were used to record the conversation, similarly to the experiment explained in Section 5.4. The experiment was conducted in the rooms where the participants reside.

The sensing result was accurate and well reflected the behaviors of the participants. The attentions of the participants were retained during the period of the agents' utterances, and the responses of the participants were mostly positive, although one of the residents gradually lost interest and positive attitude owing to shortcomings in hearing capability.

The staff opined that the volume of the sound must be adjustable and that the system must not be used with dementia patients. The overall impression was favorable because the residents appeared to be enjoying the agent's company. The results confirmed that the current system can be applied in actual care environments.

\section{Conclusion}

A bedside agent system that speaks to a person to reduce the risks of falling was developed in this study. The system monitors a person's posture and behavior, and when it senses risk, it activates simultaneously a nurse call alarm and a voice call to the person to ensure that the person stays on the bed until a caregiver arrives, thus reducing the chance of a fall.

This system was developed following the user-oriented approach, and it considers the different positions of users, including care staff (who provide care) and older adults (who are cared for).

The opinions of the care staff about the system and the reactions of the older adults to the system were considered at various stages of the development process. The knowledge gleaned from care staff pertained mainly to safety issues, and the feedback from older adults was mostly related to the contents of voice calls, which helped refine system acceptability. An overview of the process is shown in Fig. 12, and the details are summarized as follows.

First, hardware design, such as outer shape, size, and functions of the agent's mechanism, was reviewed by nurses and caregivers. Safety was the most emphasized viewpoint in this step because it is the most important issue when new equipment is introduced into a care environment. A prototype agent developed after several review processes based on user opinions was introduced into a community-dwelling of healthy older adults for experimental verification. Basic functions related to the agent's function, such as name calling and LED lighting, 
were studied in the experiment. In the development of Side-Bot, this process was repeated three times, as described in the paper.

Second, the software design of the agent, such as the contents of voice calls, was studied through multiple experiments. In this step, the major concern was acceptability of the system by real end users, namely, older adults. Two cycles of the revision processes were performed in the case of "Side-Bot".

One of the requirements discovered in this study is that an agent simply talking to a person can practically be accepted without much discomfort, and such an agent was found to be useful even without the use of speech recognition technology.

Last, an integrated model of the hardware and software was introduced to care facilities and hospitals to be used by older adults needing care. Here, system safety and acceptability were evaluated preliminarily in a real care environment. The main concern in this step was to study and ensure the practical serviceability of the system.

The staff at hospitals/care facilities recognize the system's benefits because the system has the potential to help reduce the number of fall accidents. However, the final objective, that is, system serviceability, involves i) ensuring that the patient remains on a bed longer because of the voice call of the agent, ii) confirming that the nurse can arrive at the patient's bedside while the patient remains on the bed, and iii) preventing falls as a result. The objective of system serviceability warrants further investigation.

Moreover, further developing the system by adding i) timing control capability for the agents' speech, and ii) the ability to sense a person's detailed conditions, such as sleep/awake state, should be studied to realize a more human-friendly and versatile bedside agent system.

Open Access This article is distributed under the terms of the Creative Commons Attribution 4.0 International License (http:// creativecommons.org/licenses/by/4.0/), which permits unrestricted use, distribution, and reproduction in any medium, provided you give appropriate credit to the original author(s) and the source, provide a link to the Creative Commons license, and indicate if changes were made.

\section{References}

1. Cabinet Office of Japan, Annual Report on the Aging Society (Summary) FY2016, http://www8.cao.go.jp/kourei/english/ annualreport/2016/pdf/cover.pdf (2016)

2. Augusto, J.C., Huch, M., et al.: Handbook of Ambient Assisted Living, Technology for Healthcare, Rehabilitation and Well-being. IOS Press, Washington, DC (2012)

3. Hughes, R.G. (ed.): Patient Safety and Quality: An EvidenceBased Handbook for Nurses, AHRQ Publication No. 08-0043. Agency for Healthcare Research and Quality, Rockville (2008)
4. Miyake, N., Hatsukari, T., Matsushita, S., et al.: Stand-up assist system for elderly using coordinated motion with a bed. In: 2014 Europe-Asia Congress on Mecatronics, pp. 162-167 (2014)

5. Centers for Disease Control and Prevention, WISQARS leading causes of nonfatal injury reports, http://www.cdc.gov/ncipc/ wisqars/nonfatal/quickpicks/quickpicks_2006/allinj.htm (2006)

6. Japan Council for Quality Health Care, Project to Collect Medical Near-miss/Adverse Event Information 2015 Annual Report, http:// www.med-safe.jp/pdf/year_report_english_2015.pdf (2016)

7. Carecom Co. Ltd., Mat Sensor, https://www.carecom.jp/global/ solutions/option/

8. Daielsen, A., Torresen, J.: Recognizing bedside events using thermal and ultrasonic readings. Sensors 17, 6 (2017)

9. Paramount Bed, Co. Ltd., Getting-out-of-Bed CATCH, http:// www.paramount.co.jp/contents/949

10. Miyake, N., Hatsukari, T.: Care Beds and Related Assist Systems, Instrumentation and Control, vol. 56(5), pp. 371-376. Society of Instrumentation and Control Engineers, Japan (2017)

11. Fujita, Y.: Personal Robot PaPeRo. J. Robot. Mechatron. 14(1), 60-63 (2002)

12. Wada, K., Shibata, T.: Social effects of therapy in a care house. In: Proceedings of the IEEE International Conference on Robotics and Automation, pp. 1250-1255 (2007)

13. Intuition Robotics Ltd., Elli Q, https://elliq.com/

14. Jibo Inc., Jibo, https://www.jibo.com/

15. U.S. Food and Drug Administration, Clinical Guidance For the Assessment and Implementation of Bed Rails In Hospitals, Long Term Care Facilities, and Home Care Settings, https://www.fda. gov/downloads/HospitalBeds/UCM397178.pdf

16. Hughes, R. (ed.): Rights, Risk and Restraint-Free Care of Older People: Person-Centred Approaches in Health and Social Care. Jessica Kingsley Publishers, London (2010)

17. Ministry of Health, Labor and Welfare, Physical Restraint Zero Handbook: for all the people involved in Geriatric Care, http://www.fukushihoken.metro.tokyo.jp/zaishien/gyakutai/ torikumi/doc/zero_tebiki.pdf (2001)

18. Potter, P., Grayson, D., et al.: Mapping the Nursing Process. J. Nurs. Admin. 34(2), 101-109 (2004)

19. York, T., MacAlister, D.: Hospital and Healthcare Security. Elsevier (2015)

20. Seelye, A.: Hospital ward layout and nurse staffing. J. Adv. Nurs. 7(3), 195-201 (1982)

21. Department of Education, Training and Employment, Health and Safety Risk Assessment Template, Queensland Government, Australia (2012)

22. Bruce, A., Nourbakhsh, I., Simmons, R.: The role of expressiveness and attention in human-robot interaction. Proc. IEEE Int. Conf. Robot. Automat. 4, 4138-4142 (2002)

23. Fraichard, T., Paulin, R., Reignier, P.: Human-robot motion: taking attention into account. INRIA Research Report RR-8487 (2014)

24. Schmitt, A., Minker, W.: Towards Adaptive Spoken Dialogue System. Springer, Berlin (2013)

25. Higashinaka, R., et al.: Towards an open domain conversational system fully based on natural language processing. In: Proceedings of the 25th International Conference on Computational Linguistics, pp. 928-939 (2014)

26. Yoshikawa, Y., Ito, T., Ishiguro, H., et al.: Proactive Conversation between Robots to Improve the Scene of Human-Robot Conversation, Human-Agent Groups: Studies, Algorithms, Challenges, AAAI Technical Report FS-17-04 (2017)

Publisher's Note Springer Nature remains neutral with regard to jurisdictional claims in published maps and institutional affiliations. 
Norihisa Miyake Ph.D in robotics, graduated from Graduate School of Engineering and Science, Waseda University in 1974. He is currently with RIKEN AIP (Center for Advanced Intelligence Project) since 2017. He was a Visiting Professor leading Cooperative Research Laboratory of Nursing and Engineering, Chiba University, before joining RIKEN, after he worked for Paramount Bed, Ltd. till 2013 as a chief researcher in the field of assistive robotics. He worked for Mechanical Engineering Research Laboratory, Hitachi, Ltd., where he served as a researcher, senior researcher specialized in intelligent robotics, and director of dynamics and control department respectively, since 1974 before joining Paramount Bed in 2002. He has been working in the field of robotics throughout his career. He is a fellow, Japan Society of Mechanical Engineers and a fellow, Robotics Society of Japan. (ORCID ID: 0000-0002-5715-3775)

Shouta Shibukawa MSME, graduated from Graduate School of Engineering, Chiba University in 2016. He joined Paramount Bed, Ltd., upon graduation and currently developing intelligent bed systems.
Harue Masaki RN, Ph.D in nursing, graduated from Graduate School of Nursing, Chiba University, where she has been working since her graduation. She is currently serving as a Professor of Gerontological Nursing. She is a member of Sigma Theta Tau International Honor Society of Nursing, Vice Chairperson of Japan Academy of Gerontological Nursing, Councilor of Japan Academy of Nursing Science, member of Japan Geriatrics Society, and others. Her research interests are, end-of-life care for the elderly, chronic illness nursing model and Japanese nursing sciences incorporating culturally appropriate care.

Mihoko Otake-Matsuura Ph.D in Engineering, graduated from Graduate School of Engineering, the University of Tokyo in 2003. She is currently with RIKEN AIP, leading Cognitive Behavioral Assistive Technology Team since 2017. She had been Associate Professor with Graduate School of Engineering, Chiba University until 2017, after serving as Associate Professor with Research into Artifacts, Center for Engineering, University of Tokyo from 2006 to 2012. Since 2008, she has been serving as a founding Director of NPO Fonobono Research Institute. She is a member of IEEE, SfN, RSJ, Japan Society for Artificial Intelligence and others. Her research interests are, cognitive enhancement, communication support system, health informatics and human robot interaction. 